\title{
Interference in Reading an E-Paper under Whole- Body Vibration Exposure with Subject Posture
}

\author{
M. K. Bhiwapurkar \\ Mechanical Engineering Department, IET, Mangalayatan University, Aligarh, India
}

\author{
V. H. Saran and S. P. Harsha \\ Mechanical and Industrial Engineering Department, Indian Institute of Technology, Roorkee, India
}

\begin{abstract}
There is increasing use of laptop computer in rail vehicles for performing various sedentary activities such as reading and typing. The vibration is a major factor influencing the reading performance during the journey. Therefore, an experimental study was conducted to investigate the extent of interference perceived in reading an e-paper in two seated postures (backrest support and leaning over the table) under random vibration. The study involved 30 healthy male subjects who were excited with vibration acceleration in mono-, dual, and multi-axes in 1-20 $\mathrm{Hz}$ at $0.4,0.8$, and $1.2 \mathrm{~m} / \mathrm{s}^{2}$ amplitudes. The task consisted of reading the given paragraph of an e-paper under various vibration stimuli, and it was evaluated by time taken to complete the task and subjective evaluation of reading difficulty. The subjective evaluation showed that the reading difficulty increased with vibration stimuli for both the subject postures. The subjects perceived higher difficulty and degradation in reading performance for vibration in dual and multi-axes, which was comparable to that for lateral and vertical directions also. The perceived difficulty and impairment in reading performance was greater while reading with the laptop on their lap for vibration in the $\mathrm{x}$-axis, while the effect was just the reverse for other axes.
\end{abstract}

\section{INTRODUCTION}

Today, laptop computers are widely used by commuters in rail vehicles due to the lightweight and compact size of the LCD display. During travel, the vibration environment in trains affects the human visual system and reading performance, consequently making the reading activity more difficult and tiring. A number of authors have investigated the effects of ambient luminance, light source, polarity, font style, font size, and viewing distance on visual performance and visual fatigue of e-paper displays and compared them with conventional papers, ${ }^{1-3}$ but only under stationary conditions. Khan et al. investigated the combined effect of noise and vibration on the performance of a readability task on a laptop computer under varying levels of noise and vibration. ${ }^{4,5}$ Results showed that the individual effects of noise and vibration were statistically significant. However, the combined effect of noise and vibration was not found to be statistically significant. In order to investigate the effect of train vibrations on laptop personal computers (PC) and their users, Nagakawa and Suzuki ${ }^{6}$ carried out surveys and related experiments. The results reveal that the majority of the passengers prefer to work with their laptops on their lap instead of the table. The reasons could be attributed to the inappropriate height and size of the table and as a means to attenuate the vibration.

Previous studies have confirmed that vibrations disturb a significant number of passengers in performing sedentary activities like reading. Whole-body vibration has been shown to affect both reading speed and reading accuracy in many studies. $^{7-18}$ Lewis and Griffin ${ }^{19}$ found a degradation of reading performance during exposure to fore-and-aft ( $\mathrm{x}$-axis) vibration at frequencies between 5.6 and $11 \mathrm{~Hz}$ during reading leading articles from a national newspaper. However, it was pointed out that the effect was present only when a seat with a backrest was used, and so it may be assumed that vibration transmitted to the head by the backrest was the cause of the problem. In the same study, lateral (y-axis) vibration produced only a slight degradation in reading performance at about $5.6 \mathrm{~Hz}$.

In a recent field study on various Indian Railways passenger trains, which included both questionnaire surveys and vibration measurements, it was found that the maximum difficulty was observed in writing activity, a smaller difficulty in working with laptop computers, and the comparatively smallest difficulty in reading. ${ }^{21}$ It was also revealed that most of the laptop users preferred to work with laptops on their lap instead of the table. The reasons could be attributed to the inappropriate height and size of the table and as a means to attenuate the vibration. The same study reported that the vibration acceleration levels measured from floor of passenger compartment were found to be in the range of $0.2-0.67 \mathrm{~m} / \mathrm{s}^{2}$ $\mathrm{rms}$ in the longitudinal direction (x-axis); $0.23-0.83 \mathrm{~m} / \mathrm{s}^{2} \mathrm{rms}$ in the lateral direction (y-axis) and $0.38-1.2 \mathrm{~m} / \mathrm{s}^{2} \mathrm{rms}$ in the vertical direction (z-axis). As compared with vibration acceleration in the $\mathrm{x}$-axis, the vibration acceleration level was found to be about $30 \%$ higher in the y-axis and approximately $80 \%$ higher in the z-axis. Therefore, these vibration acceleration magnitudes were considered for the study.

The objective of the study was to investigate the extent of interference perceived in reading e-papers by seated subjects in two postures under mono-, dual, and multi-axis Gaussian random vibration environment.

\section{METHODOLOGY}

\subsection{Subjects}

A total of 30 healthy male subjects with age in years $(22.91$ $\pm 4.58)$, weight in $\mathrm{kg}(68.91 \pm 12.04)$, and height in $\mathrm{cm}$ $(173.87 \pm 5.86)$, all students of the Institute with normal eyesight (normal visual acuity 6/6 vision), participated in the experiment. The subjects participated voluntarily under informed 